\title{
SOME CONJECTURES ON ENDOSCOPIC REPRESENTATIONS IN ODD ORTHOGONAL GROUPS
}

\author{
DAVID GINZBURG AND DIHUA JIANG
}

\author{
In memory of Hiroshi Saito
}

\begin{abstract}
In this paper, we introduce two conjectures on characterizations of endoscopy structures of irreducible generic cuspidal automorphic representations of odd special orthogonal groups in terms of nonvanishing of certain period of automorphic forms. We discuss a relation between the two conjectures and prove that a special case of Conjecture 1 (and hence Conjecture 2) is true.
\end{abstract}

\section{$\S 1$. Introduction}

Let $\pi$ denote an irreducible generic cuspidal automorphic representation of $\mathrm{SO}_{2 m+1}(\mathbf{A})$. Here $\mathbf{A}$ is the ring of adèles of a number field $F$. We say that $\pi$ is an endoscopic representation with respect to $\mathrm{SO}_{2 r+1} \times \mathrm{SO}_{2(m-r)+1}$ if there are generic cuspidal automorphic representations $\sigma_{1}$ and $\sigma_{2}$ of $\mathrm{SO}_{2 r+1}(\mathbf{A})$ and $\mathrm{SO}_{2(m-r)+1}(\mathbf{A})$, respectively, such that $\pi$ is the Langlands functorial lift of $\sigma_{1} \otimes \sigma_{2}$. This functorial lift corresponds to the $L$-group homomorphism $\mathrm{Sp}_{2 r}(\mathbf{C}) \times \mathrm{Sp}_{2(m-r)}(\mathbf{C}) \rightarrow \mathrm{Sp}_{2 m}(\mathbf{C})$, which is given by the direct sum embedding. More generally, we say that $\pi$ is an endoscopic representation with respect to $\mathrm{SO}_{2 r_{1}+1} \times \cdots \times \mathrm{SO}_{2 r_{k}+1}$ if there exists an irreducible generic cuspidal automorphic representation $\sigma_{i}$ of each $\mathrm{SO}_{2 r_{i}+1}(\mathbf{A})$ such that $\pi$ is the Langlands functorial lift of $\sigma_{1} \otimes \cdots \otimes \sigma_{k}$. Here $1 \leq i \leq k$ and $r_{1}+\cdots+r_{k}=m$. As above, this lift corresponds to the $L$-group homomorphism

$$
\mathrm{Sp}_{2 r_{1}}(\mathbf{C}) \times \cdots \times \mathrm{Sp}_{2 r_{k}}(\mathbf{C}) \mapsto \mathrm{Sp}_{2 m}(\mathbf{C})
$$

Received February 11, 2012. Revised September 28, 2011. Accepted May 2, 2012.

2010 Mathematics Subject Classification. Primary 11F70, 22 E55.

The work of both authors was supported in part by U.S.-Israel Binational Science Foundation grant 2008058. Jiang's work was supported in part by National Science Foundation grant DMS-1001672.

(C) 2012 by The Editorial Board of the Nagoya Mathematical Journal 
If an irreducible generic cuspidal automorphic representation $\pi$ of $\mathrm{SO}_{2 m+1}(\mathbf{A})$ is not endoscopic, we say that $\pi$ is stable (or simple, which is compatible with the notion of simple parameters of Arthur $[\mathrm{A}]$ ). It follows from the Langlands functoriality conjecture (or, more precisely, the theory of endoscopy) that, for any irreducible generic cuspidal automorphic representation $\pi$ of $\mathrm{SO}_{2 m+1}(\mathbf{A})$, there exists a partition $m=\sum_{i=1}^{k} r_{i}$ and there exists an irreducible, stable, generic cuspidal automorphic representation $\sigma_{i}$ of each $\mathrm{SO}_{2 r_{i}+1}(\mathbf{A})$ such that $\pi$ is an endoscopy lift from $\sigma_{1} \otimes \cdots \otimes \sigma_{k}$. In this case, we say that $\pi$ has a stable endoscopy type $\left(\sigma_{1}, \ldots, \sigma_{k}\right)$. Hence, if $\pi$ has a stable endoscopy type $\left(\sigma_{1}, \ldots, \sigma_{k}\right)$, then $\pi$ is also endoscopic with respect to $\mathrm{SO}_{2 r+1} \times \mathrm{SO}_{2(m-r)+1}$ for a suitable value of $r$.

This suggests two important problems.

(1) Existence. Given a set of irreducible cuspidal automorphic representations $\sigma_{i}$ defined on $\mathrm{SO}_{2 r_{i}+1}(\mathbf{A})$, with $i=1,2, \ldots, k$, prove the existence of an endoscopic representation $\pi$ of $\mathrm{SO}_{2 m+1}(\mathbf{A})$ with respect to $\mathrm{SO}_{2 r_{1}+1} \times \cdots \times \mathrm{SO}_{2 r_{k}+1}$, which is the endoscopic transfer from $\sigma_{1} \otimes \cdots \otimes \sigma_{k}$.

(2) Characterization. Characterize the image of this lift. In other words, given an irreducible generic cuspidal automorphic representation $\pi$ of $\mathrm{SO}_{2 m+1}(\mathbf{A})$, determine when it is an endoscopic representation. This characterization can be given in terms of poles of $L$-functions, of nonvanishing of certain period integrals, or of both.

For problem (1), currently there are constructive methods to prove the existence of such lifts. The first construction is the so-called descent method. This method uses a Fourier coefficient of a certain residue of an Eisenstein series defined on the even orthogonal groups (see [S] for some details). Another construction of endoscopic representation is given in [G3], using an extension of the descent method. We should mention that both constructions use the functorial lift from an odd orthogonal group to $\mathrm{GL}_{n}$ as established in [CKPS]. The existence of such endoscopy lifts is in general expected to follow from the method of the trace formula.

For the characterization of the image, that is, problem (2), some partial results are known. First, regarding poles of $L$-functions, the result of $[\mathrm{J}]$ gives a relation between this lifting and the order of the pole of the partial $L$-function attached to the second fundamental representation of $\operatorname{Sp}_{2 m}(\mathbf{C})$. Regarding the characterization of the image of the lift in terms of period integrals, we have the following. 
Theorem 1 ([G3, Theorem 6]). An irreducible generic cuspidal automorphic representation $\pi$ of $\mathrm{SO}_{2 m+1}(\mathbf{A})$ has a stable endoscopy type $\left(\sigma_{1}, \ldots, \sigma_{k}\right)$, with $\sigma_{i}$ being an irreducible stable generic cuspidal automorphic representation of $\mathrm{SO}_{2 r_{i}+1}(\mathbf{A}), i=1,2, \ldots, k$, if and only if there exist $k$ irreducible, nonisomorphic, cuspidal automorphic representations $\tau_{i}$ of $\mathrm{GL}_{2 r_{i}}(\mathbf{A})$, respectively, such that all the period integrals $\mathcal{P}\left(\pi, \tau_{i}\right)$, defined in [G3], are nonzero for some choice of data.

Even though the above theorem does give a characterization of the image, there are two problems with it. First, it requires a set of period integrals and not just one. Second, the period integral is not defined using the representation $\pi$ only but also in terms of cuspidal representations of the group $\mathrm{GL}_{n}(\mathbf{A})$.

In this paper we introduce a family of period integrals which we conjecture to give a certain characterization of the image of the lift. Our main conjecture is the following.

Conjecture 1. Assume that $2 r \leq m$. An irreducible generic cuspidal automorphic representation $\pi$ is endoscopic with respect to $\mathrm{SO}_{2 r+1} \times$ $\mathrm{SO}_{2(m-r)+1}$ if and only if the period integral

$$
\mathcal{Q}_{r}(\pi)=\int_{\operatorname{Sp}_{2 r}(F) \backslash \mathrm{Sp}_{2 r}(\mathbf{A})} \int_{U_{r}(F) \backslash U_{r}(\mathbf{A})} \varphi_{\pi}(u h) \psi_{U_{r}}(u) d u d h
$$

is not 0 for some choice of data.

The precise definition of the integrals $\mathcal{Q}_{r}(\pi)$ will be given in Section 2. The difference between Conjecture 1 and Theorem 1 is that in Conjecture 1 , the period integral involves only the representation $\pi$ and only one period integral. In contrast, Theorem 1 is more general and also gives the stable endoscopy type of $\pi$.

The main result related to this conjecture is as follows.

Theorem 2. Conjecture 1 holds for $r=1$.

We prove this theorem in Section 2.

In the third section we state a conjecture involving a period integral, which characterizes when the representation $\pi$ is an endoscopic functorial lift from $\mathrm{SO}_{3} \times \mathrm{SO}_{2 r+1} \times \mathrm{SO}_{2(m-r)-1}$. Clearly, if we use Theorem 1 to this case, we need three different but similar types of periods to characterize this endoscopy structure (i.e., three endoscopy factors). Our conjecture (Conjecture 2 in Section 3) characterizes the endoscopy structure (with three 
endoscopy factors) in terms of one period integral. However, in contrast to Conjecture 1, the period integral used in Conjecture 2 not only involves the representation $\pi$ but also involves a cuspidal representation of $\mathrm{SO}_{3}(\mathbf{A})$. The main result in Section 3 is to prove that Conjecture 2 follows from Conjecture 1, in particular, that Conjecture 2 holds for $r=1$.

The last section is an appendix where we collect some known results which we use mainly in the third section. We also remark that the main idea used in the proofs in this paper is to consider various Fourier expansions of automorphic forms along certain unipotent subgroups. In general, one may consider Fourier coefficients of automorphic forms attached to unipotent orbits. We refer to [G2] and the references therein for general discussions on this topic.

\section{$\S 2$. On the main conjecture}

We keep the notation of the introduction. Let $\pi$ denote an irreducible generic cuspidal automorphic representation of $\mathrm{SO}_{2 m+1}(\mathbf{A})$. The orthogonal group is realized as the group of all matrices $g \in \mathrm{GL}_{2 m+1}$ such that $g^{t} J g=J$. Here $J$ is the $2 m+1$ matrix which has ones on the other diagonal and zeros elsewhere.

We now describe the groups used to define the period integral $\mathcal{Q}_{r}(\pi)$ as given in integral (1). Here $2 r \leq m$. Let $U_{r}^{\prime}$ denote the unipotent radical of the parabolic subgroup of $\mathrm{SO}_{2 m+1}$ whose Levi part is $\mathrm{GL}_{1}^{m-2 r} \times \mathrm{GL}_{2 r}$. Let $U_{r}$ be the subgroup of $U_{r}^{\prime}$ which consists of all $u=\left(u_{i, j}\right) \in U_{r}^{\prime}$ such that $u_{i, m+1}=0$ for all $m-2 r+1 \leq i \leq m$. Let $\psi$ denote a nontrivial additive character of $F \backslash \mathbf{A}$. The character $\psi_{U_{r}}$ is defined as

$$
\begin{aligned}
\psi_{U_{r}}(u)= & \psi\left(u_{1,2}+u_{2,3}+\cdots+u_{m-2 r-1, m-2 r}+u_{m-2 r, m+1}\right. \\
& \left.+u_{m-2 r+1, m+2}+u_{m-2 r+2, m+3}+\cdots+u_{m-r, m+r+1}\right) .
\end{aligned}
$$

Then $\mathrm{Sp}_{2 r}$ embedded inside $\mathrm{GL}_{2 r}$ stabilizes the character $\psi_{U_{r}}$. We note that for experienced readers it will not be hard to find that the introduction of the unipotent subgroup $U_{r}$ and the character $\psi_{U_{r}}$ is motivated by the automorphic descent constructions and generalized Shalika periods (see [GRS], [JQ]).

Proof of Theorem 2. Assume first that $\pi$ is endoscopic with respect to $\mathrm{SO}_{3} \times \mathrm{SO}_{2 m-1}$. Thus, $\pi$ is the functorial lift of an irreducible generic cuspidal automorphic representation $\tau$ of $\mathrm{SO}_{3}(\mathbf{A})$ and an irreducible generic cuspidal 
automorphic representation $\sigma$ of $\mathrm{SO}_{2 m-1}(\mathbf{A})$. We view $\tau$ as an irreducible representation of $\mathrm{GL}_{2}(\mathbf{A})$ with a trivial central character. Then, as stated in Theorem 1, it follows from [G3] that the integral

$$
\int_{\mathrm{SO}_{4}(F) \backslash \mathrm{SO}_{4}(\mathbf{A})} \int_{V_{1}(F) \backslash V_{1}(\mathbf{A})} \varphi_{\pi}(v g) \psi_{U_{1}}(v) E_{\tau}(g) d v d g
$$

is not 0 for some choice of data. Here $V_{1}$ is the unipotent radical of the parabolic subgroup of $\mathrm{SO}_{2 m+1}$ whose Levi part is $\mathrm{GL}_{1}^{m-2} \times \mathrm{SO}_{5}$. Notice that $V_{1}$ is a subgroup of $U_{1}$, and we view $\psi_{U_{1}}$ as a character of $V_{1}$ by restriction. Also, $E_{\tau}(g)$ denotes the residue of the Eisenstein series defined on $\mathrm{SO}_{4}$ which is induced from $\tau$. More precisely, let $E_{\tau}(g, s)$ denote the Eisenstein series of $\mathrm{SO}_{4}(\mathbf{A})$ associated with the induced representation $\operatorname{Ind}_{P_{2}(\mathbf{A})}^{\mathrm{SO}_{4}(\mathbf{A})} \tau \delta_{P_{2}}^{s}$. Here $P_{2}$ is a maximal parabolic subgroup of $\mathrm{SO}_{4}$ whose Levi part is $\mathrm{GL}_{2}$. Since $\tau$ has a trivial central character, this Eisenstein series has a simple pole at $s=1$. We denote the residue representation by $E_{\tau}(g)$.

Restricting $E_{\tau}(g)$ as a function to $\mathrm{SL}_{2}(\mathbf{A}) \times \mathrm{SL}_{2}(\mathbf{A})$, it follows from integral (2) that the integral

$$
\begin{aligned}
& \int_{\left(\mathrm{SL}_{2}(F) \times \mathrm{SL}_{2}(F)\right) \backslash\left(\mathrm{SL}_{2}(\mathbf{A}) \times \mathrm{SL}_{2}(\mathbf{A})\right)} \int_{V_{1}(F) \backslash V_{1}(\mathbf{A})} \varphi_{\pi}\left(v\left(g_{1}, g_{2}\right)\right) \\
& \quad \times \psi_{U_{1}}(v) \varphi_{\tau}\left(g_{1}\right) d v d g_{1} d g_{2}
\end{aligned}
$$

is not 0 for some choice of data. This implies that as a function of $g_{1} \in$ $\mathrm{SL}_{2}(\mathbf{A})$, the function

$$
f\left(g_{1}\right)=\int_{\mathrm{SL}_{2}(F) \backslash \mathrm{SL}_{2}(\mathbf{A})} \int_{V_{1}(F) \backslash V_{1}(\mathbf{A})} \varphi_{\pi}\left(v\left(g_{1}, g_{2}\right)\right) \psi_{U_{1}}(v) d v d g_{2}
$$

is not 0 , and moreover, it is not the identity function. Therefore, it has a nonzero Fourier coefficient. Thus, there is $\alpha \in F^{*}$ such that the integral

$$
\begin{aligned}
& \int_{\mathrm{SL}_{2}(F) \backslash \mathrm{SL}_{2}(\mathbf{A})} \int_{F \backslash \mathbf{A}} \int_{V_{1}(F) \backslash V_{1}(\mathbf{A})} \varphi_{\pi}\left(v\left(\left(\begin{array}{ll}
1 & x \\
& 1
\end{array}\right) g_{1}, g_{2}\right)\right) \\
& \times \psi(\alpha x) \psi_{U_{1}}(v) d x d v d g_{2}
\end{aligned}
$$

is not 0 for some choice of data. However, conjugating by the torus element of $\mathrm{SO}_{2 m+1}$ given by $\operatorname{diag}\left(I_{m_{2}}, \alpha, I_{3}, \alpha^{-1}, I_{m-2}\right)$, we deduce that when $\alpha=1$, the above integral is not 0 for some choice of data. By definition, the unipotent group we integrate over in the above integral is the group $U_{1}$. Also, the 
character defined on this group is exactly $\psi_{U_{1}}$. Thus, we conclude that integral (1) with $r=1$ is not 0 for some choice of data.

To prove the converse, we start with integral (1), and arguing backward, we deduce that integral (3) is not 0 for some choice of data. Changing variables in the $g_{2}$ variable in integral $(3)$, we obtain that $f\left(\left(\begin{array}{c}\alpha_{1} \\ { }_{1}\end{array}\right) g_{1}\right)=$ $f\left(g_{1}\right)$ for all $\alpha \in F^{*}$ and that $f\left(\left(\begin{array}{c}a_{a} \\ a\end{array}\right) g_{1}\right)=f\left(g_{1}\right)$ for all $a \in \mathbf{A}^{*}$. Hence, the function $f\left(g_{1}\right)$ defined in integral (3) defines a nonzero automorphic function of $\mathrm{GL}_{2}(\mathbf{A})$. Let $\tau^{\prime}$ denote the representation of $\mathrm{GL}_{2}(\mathbf{A})$ generated by the space of functions $f\left(g_{1}\right)$ given by $(3)$. It follows from the above that $\tau^{\prime}$ has a trivial central character. We claim that $\tau^{\prime}$ is a cuspidal representation. Assuming that, let $\tau$ denote an irreducible summand of $\tau^{\prime}$. From the above, we deduce that the integral

$$
\begin{aligned}
& \int_{Z(\mathbf{A}) \mathrm{GL}_{2}(F) \backslash \mathrm{GL}_{2}(\mathbf{A})} \int_{\left.\mathrm{SL}_{2}(F)\right) \backslash \mathrm{SL}_{2}(\mathbf{A})} \int_{V_{1}(F) \backslash V_{1}(\mathbf{A})} \varphi_{\pi}\left(v\left(g_{1}, g_{2}\right)\right) \\
& \times \psi_{U_{1}}(v) \varphi_{\tau}\left(g_{1}\right) d v d g_{1} d g_{2}
\end{aligned}
$$

is not 0 for some choice of data, which implies that the integral (2) is not 0 for some choice of data. Thus, it follows from [G3] that $\pi$ is endoscopic.

It remains to prove that the representation $\tau^{\prime}$ is cuspidal. Thus, we need to prove that the integral

$$
\int_{F \backslash \mathbf{A}} f\left(\left(\begin{array}{ll}
1 & x \\
& 1
\end{array}\right) g_{1}\right) d x
$$

is 0 for all choice of data. Let

$$
w=\left(\begin{array}{ccccc} 
& I_{2} & & & \\
I_{m-2} & & & & \\
& & 1 & & \\
& & & I_{m-2}
\end{array}\right) .
$$

Conjugating the argument of $\varphi_{\pi}$ by $w$, we need to prove that the integral

$$
\begin{aligned}
\int \varphi_{\pi} & {\left[\left(\begin{array}{ccc}
I_{2} & x & y \\
& I_{2 m-3} & x^{*} \\
& & I_{2}
\end{array}\right)\left(\begin{array}{ccc}
I_{2} & & \\
& v & \\
& & I_{2}
\end{array}\right)\right.} \\
& \left.\times\left(\begin{array}{ccc}
I_{2} & & \\
z & I_{2 m-3} & \\
& z^{*} & I_{2}
\end{array}\right)\left(\begin{array}{ccc}
g & & \\
& I_{2 m-3} & \\
& & g^{*}
\end{array}\right)\right] \psi_{V}(v) d(\cdots)
\end{aligned}
$$


is 0 for all choice of data. Here, $x \in \mathrm{Mat}_{2 \times(2 m-3)}^{f, m-1}$ is integrated over the group of matrices $\operatorname{Mat}_{2 \times(2 m-3)}^{f, m-1}(F) \backslash \operatorname{Mat}_{2 \times(2 m-3)}^{f, m-1}(\mathbf{A})$, where $f, m-1$ indicate that the first $m-1$ columns are 0 . Also, $y$ is a $2 \times 2$ matrix such that the first matrix in the argument of $\varphi_{\pi}$ in integral (4) is a matrix in $\mathrm{SO}_{2 m+1}$. Let $V$ denote the maximal unipotent subgroup of $\mathrm{SO}_{2 m-3}$. We embed it inside $\mathrm{SO}_{2 m+1}$ as in the second matrix in integral (4). Then, $v$ is integrated over $V(F) \backslash V(\mathbf{A})$. The character $\psi_{V}$ is the Whittaker character of $V$. In other words, if $v=\left(v_{i, j}\right) \in V$, then $\psi_{V}(v)=\psi\left(v_{1,2}+v_{2,3}+\cdots+v_{m-2, m-1}\right)$. Next, $z$ is integrated over $\mathrm{Mat}_{2 \times(2 m-3)}^{l, m-1}(F) \backslash \mathrm{Mat}_{2 \times(2 m-3)}^{l, m-1}(\mathbf{A})$, where $l, m-1$ indicate that the last $m-1$ rows are 0 . Finally, $g$ is integrated over $\mathrm{SL}_{2}(F) \backslash \mathrm{SL}_{2}(\mathbf{A})$.

Next, we perform a sequence of Fourier expansions. We start by expanding integral (4) along the unipotent group $l\left(r_{1}, r_{2}\right)=I_{2 m+1}+r_{1}\left(e_{1, m+1}-\right.$ $\left.e_{m+1,2 m+1}\right)+r_{1}^{2} e_{1,2 m+1}+r_{2}\left(e_{2, m+1}-e_{m+1,2 m}\right)+r_{2}^{2} e_{2,2 m}$. Here, for all $i, j$, we denote by $e_{i, j}$ the $(2 m+1) \times(2 m+1)$ matrix which has 1 in the $(i, j)$ entry and 0 elsewhere. Thus, integral (4) is equal to

$$
\sum_{\alpha_{1}, \alpha_{2} \in F} \int \varphi_{\pi}\left(l\left(r_{1}, r_{2}\right)(x, y, v, z, g)\right) \psi_{V}(v) \psi\left(\alpha_{1} r_{1}+\alpha_{2} r_{2}\right) d(\cdots) .
$$

Here, $r_{1}$ and $r_{2}$ are integrated over $F \backslash \mathbf{A}$ and all other variables are integrated as before. For fixed $\alpha_{1}, \alpha_{2} \in F$, consider the matrix $s\left(\alpha_{1}, \alpha_{2}\right)=$ $I_{2 m+1}+\alpha_{1}\left(e_{m, 1}-e_{2 m+1, m+2}\right)+\alpha_{2}\left(e_{m, 2}-e_{2 m, m+2}\right)$. Use the left invariant property of $\varphi_{\pi}$ under matrices in $\mathrm{SO}_{2 m+1}(F)$, to write in the above integral $\varphi_{\pi}(h)=\varphi_{\pi}\left(s\left(\alpha_{1}, \alpha_{2}\right) h\right)$. Conjugating $s\left(\alpha_{1}, \alpha_{2}\right)$ to the right, changing variables in $v$, we obtain that the above integral is equal to

$$
\int \varphi_{\pi}((x, y, v, z, g)) \psi_{V}(v) d(\cdots)
$$

where now $x$ is integrated over $\operatorname{Mat}_{2 \times(2 m-3)}^{f, m-2}(F) \backslash \operatorname{Mat}_{2 \times(2 m-3)}^{f, m-2}(\mathbf{A})$ and $z$ is integrated over $\mathrm{Mat}_{2 \times(2 m-3)}^{l, m-2}(F) \backslash \mathrm{Mat}_{2 \times(2 m-3)}^{l, m-1}(\mathbf{A})$. All other variables are integrated as before. We continue this process, and we deduce that the following integral

$$
\int \varphi_{\pi}\left[\left(\begin{array}{ccc}
I_{2} & x & y \\
& I_{2 m-3} & x^{*} \\
& & I_{2}
\end{array}\right)\left(\begin{array}{ccc}
I_{2} & & \\
& v & \\
& & I_{2}
\end{array}\right)\left(\begin{array}{ccc}
g & & \\
& I_{2 m-3} & \\
& & g^{*}
\end{array}\right)\right] \psi_{V}(v) d(\cdots)
$$

is an inner integration to the integral (4). Here, $x$ is integrated over the group of matrices $\mathrm{Mat}_{2 \times(2 m-3)}^{f, 1}(F) \backslash \operatorname{Mat}_{2 \times(2 m-3)}^{f, 1}(\mathbf{A})$ and all other variables 
are integrated as before. Thus, it is enough to show that this integral is 0 for all choices of data. To do that, we expand it along the group $l\left(r_{1}, r_{2}\right)=$ $I_{2 m+1}+r_{1}\left(e_{1,3}-e_{2 m-1,2 m+1}\right)+r_{2}\left(e_{2,3}-e_{2 m-1,2 m}\right)$. Thus, the above integral is equal to

$$
\sum_{\alpha_{1}, \alpha_{2} \in F} \int \varphi_{\pi}\left(l\left(r_{1}, r_{2}\right)(x, y, v, g)\right) \psi_{V}(v) \psi\left(\alpha_{1} r_{1}+\alpha_{2} r_{2}\right) d(\cdots)
$$

Recall that $g$ is integrated along $\mathrm{SL}_{2}(F) \backslash \mathrm{SL}_{2}(\mathbf{A})$. From the embedding of this group inside $\mathrm{SO}_{2 m+1}$, as described in integral (4), it follows that this group acts on the above expansion with two orbits. The trivial orbit contributes 0 to the above integral. Indeed, in this case, the integration over $x$ and $y$ produces the integral of $\varphi_{\pi}$ along the unipotent radical of the maximal parabolic subgroup of $\mathrm{SO}_{2 m+1}$ whose Levi part is $\mathrm{GL}_{2} \times \mathrm{SO}_{2 m-3}$. By the cuspidality of $\pi$, it follows that it is 0 . When considering the second orbit of the above expansion, the stabilizer inside $\mathrm{SL}_{2}$ is the group $N$ which consists of all upper unipotent matrices inside $\mathrm{SL}_{2}$. If we combine this group with the $x$ and $y$ integration, we obtain as inner integration an integration over a unipotent radical of a parabolic subgroup of $\mathrm{SO}_{2 m+1}$. This time it is the parabolic subgroup whose Levi part is $\mathrm{GL}_{1} \times \mathrm{SO}_{2 m-1}$. Thus, it is also 0 . Thus, integral (4) is 0 which implies that the representation $\tau^{\prime}$ is cuspidal.

The proof of Theorem 2 is complete.

\section{$\S 3$. On the lift from three orthogonal groups}

Let $1 \leq r$, and assume that $m \geq 2 r+1$. In this section, we state our conjecture on when an irreducible generic cuspidal automorphic representation $\pi$ of $\mathrm{SO}_{2 m+1}(\mathbf{A})$ is an endoscopic representation with respect to $\mathrm{SO}_{3} \times \mathrm{SO}_{2 r+1} \times \mathrm{SO}_{2(m-r)-1}$. Notice that since $m \geq 2 r+1$, then $2(m-r)-$ $1 \geq 2 r+1$.

To state the conjecture, we first fix some notation. Let $E(g, s)$ denote the Eisenstein series on $\mathrm{SO}_{4(r+1)}$ associated with the induced representation $\operatorname{Ind}_{P(\mathbf{A})}^{\mathrm{SO}_{4(r+1)}(\mathbf{A})} \delta_{P}^{s}$. Here $P$ is the maximal parabolic subgroup of $\mathrm{SO}_{4(r+1)}$ whose Levi part is $\mathrm{GL}_{2(r+1)}$. For $\operatorname{Re}(s)>1 / 2$, the poles of this Eisenstein series were studied in $[\mathrm{KR}]$. Let $\Theta_{\left[2^{2 r} 1^{4}\right]}$ denote the residual representation of $E(g, s)$ at the point $s_{0}=(r+1) /(2 r+1)$. Then one can show that the unipotent orbit attached to this residual representation is $\left[2^{2 r} 1^{4}\right]$. 
With the above data, we define the following family of period integrals. First, if $m=2 r+1$, define

$$
\mathcal{P}_{r}(\pi, \tau)=\int_{\mathrm{SO}_{4 r+3}(F) \backslash \mathrm{SO}_{4 r+3}(\mathbf{A})} \varphi_{\pi}(g) \theta_{\tau, 4(r+1)}(g) \theta_{\left[2^{2 r} 1^{4}\right]}(g) d g .
$$

Here $\theta_{\left[2^{2 r} 1^{4}\right]}$ is a vector in the representation $\Theta_{\left[2^{2 r} 1^{4}\right]}$, and $\theta_{\tau, 4(r+1)}$ is a vector in the residual representation $\Theta_{\tau, 4(r+1)}$, as defined in [G3].

When $m>2 r+1$, we define $\mathcal{P}_{r}(\pi, \tau)$ to be equal to

$$
\begin{gathered}
\int_{\mathrm{SO}_{4(r+1)}(F) \backslash \mathrm{SO}_{4(r+1)}(\mathbf{A})} \int_{V_{m-2 r-2}^{m}(F) \backslash V_{m-2 r-2}^{m}(\mathbf{A})} \varphi_{\pi}(v g) \\
\times \psi_{V_{m-2 r-2}^{m}}(v) \theta_{\tau, 4(r+1)}(g) \theta_{\left[2^{2 r} 1^{4}\right]}(g) d v d g .
\end{gathered}
$$

Here, for $1 \leq k \leq m$, we denote by $V_{k}^{m}$ the unipotent subgroup of $\mathrm{SO}_{2 m+1}$ defined as follows. Consider the standard parabolic subgroup of $\mathrm{SO}_{2 m+1}$ whose Levi part is $\mathrm{GL}_{1}^{k} \times \mathrm{SO}_{2 m-2 k+1}$. We denote its unipotent radical by $V_{k}^{m}$. To define $\psi_{V_{k}^{m}}$, let $v=\left(v_{i, j}\right) \in V_{k}^{m}$. Then we set $\psi_{V_{k}}(v)=\psi\left(v_{1,2}+v_{2,3}+\right.$ $\left.\cdots+v_{k-1, k}+v_{k, m+1}\right)$. We have the following.

CONJECTURE 2. With the above notation, the representation $\pi$ is endoscopic with respect to $\mathrm{SO}_{3} \times \mathrm{SO}_{2 r+1} \times \mathrm{SO}_{2(m-r)-1}$ if and only if the period integral $\mathcal{P}_{r}(\pi, \tau)$ is not 0 for some choice of data.

The relation between the two conjectures is as follows.

Theorem 3. Conjecture 1 implies Conjecture 2.

Corollary 1. Conjecture 2 holds for $r=1$.

\subsection{Proof of Theorem 3}

Assume first that $\pi$ is endoscopic with respect to $\mathrm{SO}_{3} \times \mathrm{SO}_{2 r+1} \times$ $\mathrm{SO}_{2(m-r)-1}$. Thus, there exist irreducible generic cuspidal automorphic representations $\tau, \mu$, and $\epsilon$ of $\mathrm{SO}_{3}(\mathbf{A}), \mathrm{SO}_{2 r+1}(\mathbf{A})$, and $\mathrm{SO}_{2(m-r)-1}(\mathbf{A})$, respectively, such that $\pi$ is endoscopic with respect to $(\tau, \mu, \epsilon)$.

Consider the automorphic function on $\mathrm{SO}_{2 m-1}(\mathbf{A})$ defined by integral (26) in the appendix, and let $\sigma$ denote the representation of $\mathrm{SO}_{2 m-1}(\mathbf{A})$ generated by these functions. Since $\pi$ is endoscopic with respect to $(\tau, \mu, \epsilon)$, it follows from Theorem 1 that integral (2) is not 0 for some choice of data. Applying Theorem 5 in the appendix, we deduce that $\sigma$ is a nonzero generic 
cuspidal representation. Moreover, it is endoscopic with respect to $(\mu, \epsilon)$ as defined above. Since we assume Conjecture 1, we deduce that the integral

$$
\mathcal{Q}_{r}(\sigma)=\int_{\operatorname{Sp}_{2 r}(F) \backslash \operatorname{Sp}_{2 r}(\mathbf{A})} \int_{U_{r}^{m-1}(F) \backslash U_{r}^{m-1}(\mathbf{A})} \varphi_{\sigma}(u h) \psi_{U_{r}^{m-1}}(u) d u d h
$$

is not 0 for some choice of data. Notice that in integral (1) the representation $\pi$ is defined on $\mathrm{SO}_{2 m+1}$, and in integral (5) the representation $\sigma$ is defined on $\mathrm{SO}_{2 m-1}$. Also, we wrote $U_{r}^{m-1}$ in integral (5) instead of $U_{r}$ as written in integral (1). This means that here we view the groups $\operatorname{Sp}_{2 r}$ and $U_{r}^{m-1}$ as subgroups of $\mathrm{SO}_{2 m-1}$. Plugging integral (26) in the appendix into integral (5), we deduce that the integral

$$
\begin{aligned}
& \int_{\mathrm{Sp}_{2 r}(F) \backslash \mathrm{Sp}_{2 r}(\mathbf{A})} \int_{U_{r}^{m-1}(F) \backslash U_{r}^{m-1}(\mathbf{A})} \int_{\mathrm{SO}_{2 m+1}(F) \backslash \mathrm{SO}_{2 m+1}(\mathbf{A})} \varphi_{\pi}(g) \\
& \quad \times \theta_{\tau, 4 m}((g, u h)) \psi_{U_{r}^{m-1}}(u) d g d u d h
\end{aligned}
$$

is not 0 for some choice of data. This integral converges, but not absolutely. In fact, because $\sigma$ is a cuspidal representation, it follows that the integral

$$
\begin{aligned}
& \int_{\mathrm{Sp}_{2 r}(F) \backslash \mathrm{Sp}_{2 r}(\mathbf{A})} \mid \int_{U_{r}^{m-1}(F) \backslash U_{r}^{m-1}(\mathbf{A})} \int_{\mathrm{SO}_{2 m+1}(F) \backslash \mathrm{SO}_{2 m+1}(\mathbf{A})} \varphi_{\pi}(g) \\
& \quad \times \theta_{\tau, 4 m}((g, u h)) \psi_{U_{r}^{m-1}}(u) d g d u \mid d h
\end{aligned}
$$

converges. Thus, as long as we do not change the order of integration involving the $h$ variable, we can perform a series of Fourier expansions.

The embedding of the matrices $(g, u h)$ inside $\mathrm{SO}_{4 m}$ is as follows. First we embed

$$
(g, h) \mapsto \operatorname{diag}\left(I_{m-2 r-1}, h, g, h^{*}, I_{m-2 r-1}\right) .
$$

Then, the embedding of the group $U_{r}^{m-1}$ is as follows:

$$
u=\left(\begin{array}{ccccc}
u_{1} & u_{2} & u_{3} & u_{4} & u_{5} \\
& I_{2 r} & & y & u_{4}^{*} \\
& & 1 & & u_{3}^{*} \\
& & & I_{2 r} & u_{2}^{*} \\
& & & & u_{1}^{*}
\end{array}\right) \mapsto(1, u)
$$




$$
=\left(\begin{array}{ccccccc}
u_{1} & u_{2} & 0 & u_{3} & 0 & u_{4} & u_{5} \\
& I_{2 r} & 0 & 0 & 0 & y & u_{4}^{*} \\
& & I_{2 m} & & & 0 & 0 \\
& & & I_{2} & & 0 & u_{3}^{*} \\
& & & & I_{2 m} & 0 & 0 \\
& & & & & I_{2 r} & u_{2}^{*} \\
& & & & & & u_{1}^{*}
\end{array}\right) .
$$

Here, the variables are defined as follows. First, we have $u_{1} \in Z_{m-2 r-1}$ defined to be the standard maximal unipotent subgroup of $\mathrm{GL}_{m-2 r-1}$. Thus, $Z_{m-2 r-1}$ consists of all upper unipotent matrices. Next, we have $u_{2} \in$ $\operatorname{Mat}_{(m-2 r-1) \times 2 r}$ and $u_{3} \in \operatorname{Mat}_{(m-2 r-1) \times 1}$. The matrix $\left(\begin{array}{cc}u_{4} & u_{5} \\ y & u_{4}^{*}\end{array}\right) \in$ $\operatorname{Mat}_{(m-1) \times(m-1)}$ is such that the above matrix $u$ is in $\mathrm{SO}_{2 m-1}$. Finally, all $u_{i}^{*}$ are such that the above matrix is in $\mathrm{SO}_{4 m}$.

To proceed, we will apply an inductive argument. In other words, we will relate integral (6) for the values $(m, r)$ when $m>2 r+1$ with integral (6) for the values $(m-1, r)$.

Let $Y_{1}$ denote the unipotent subgroup of $\mathrm{SO}_{4 m}$ which consists of all matrices of the form

$$
y_{1}=I_{4 m}+\sum_{i=1}^{2 m+2}\left[r_{i}\left(e_{1, m+i-1}-e_{m+i-1,4 m}\right)\right]+r^{*} e_{1,4 m} .
$$

Here $r^{*}$, which depends on $r_{1}, \ldots, r_{2 m+2}$, is such that the above matrix is in $\mathrm{SO}_{4 m}$. Expand the function $\theta_{\tau, 4 m}$ in integral (6) along the group $Y_{1}(F) \backslash Y_{1}(\mathbf{A})$. The group $\mathrm{SO}_{2 m+2}$, embedded in $\mathrm{SO}_{4 m}$ as $t \mapsto \operatorname{diag}\left(I_{m-1}\right.$, $\left.t, I_{m-1}\right)$, acts on this expansion with three type of orbits. Each term of the expansion corresponds to a vector of size $2 m+2$. Consider the contribution to the expansion from the orbits corresponding to vectors of nonzero length. For $1 \leq i \leq 2 m-1$, let $L_{i}$ denote the unipotent radical of the standard parabolic subgroup of $\mathrm{SO}_{4 m}$ whose Levi part is $\mathrm{GL}_{1}^{i} \times \mathrm{SO}_{2(2 m-i)}$. Thus, when considering, in the above expansion, the terms corresponding to vectors with nonzero length, we get, when combining the integration over $Y_{1}$ and $U_{r}^{m-1}$, the integral

$$
\int_{L_{1}(F) \backslash L_{1}(\mathbf{A})} \theta_{\tau, 4 m}(l) \psi_{L}(l) d l
$$

as inner integration. Here $\psi_{L}$ is defined as follows. For $l=\left(l_{i, j}\right)$, we have $\psi_{L}(l)=\psi\left(l_{1,2}+l_{1,2 m}+\alpha l_{1,2 m+1}\right)$ for some $\alpha \in F^{*}$. It follows from the 
description of Fourier coefficients corresponding to unipotent orbits as described in [G2] that this Fourier coefficient corresponds to the unipotent orbit with partition $\left(31^{4 m-3}\right)$. From Proposition 1 in the appendix, it follows that the representation $\Theta_{\tau, 4 m}$ does not support this Fourier coefficient. Thus, the contribution to the expansion from vectors of nonzero length is 0 .

Similarly, the contribution to the expansion from the constant term along $Y_{1}$ also contributes 0 . To see that we further expand the integral, this time along the unipotent group $Y_{2}$ given by

$$
y_{2}=I_{4 m}+\sum_{i=1}^{2 m+2}\left[r_{i}\left(e_{2, m+i-1}-e_{m+i-1,4 m-1}\right)\right]+r^{*} e_{2,4 m-1} .
$$

Acting on this expansion by the same copy of $\mathrm{SO}_{2 m+2}$ we obtain two types of Fourier coefficients. The first type corresponds to the unipotent orbit with partition $\left(51^{4 m-5}\right)$ which contributes 0 by Proposition 1 in the appendix. In the second type of Fourier coefficient, we obtain the integral

$$
\int_{L_{2}(F) \backslash L_{2}(\mathbf{A})} \theta_{\tau, 4 m}(l) \psi_{L_{2}}(l) d l
$$

as inner integration. Here $\psi_{L_{2}}$ is defined as follows. For $l=\left(l_{i, j}\right)$, we have $\psi_{L_{2}}(l)=\psi\left(l_{1,2}+l_{2,3}\right)$. Further expansions show that $\Theta_{\tau, 4 m}$ cannot support this Fourier coefficient.

Thus, in the expansion along $Y_{1}$, we are left with the term corresponding to nonzero vectors of 0 length. Under the action of $\mathrm{SO}_{2 m+2}(F)$, this is one orbit, and hence integral (6) is equal to

$$
\begin{gathered}
\sum_{\gamma \in P^{0}\left(\mathrm{SO}_{2 m}\right)(F) \backslash \mathrm{SO}_{2 m+2}(F)} \int_{Y_{1}(F) \backslash Y_{1}(\mathbf{A})} \varphi_{\pi}(g) \\
\times \theta_{\tau, 4 m}\left(y_{1} \gamma(g, u h)\right) \psi_{Y_{1}}\left(y_{1}\right) \psi_{U_{r}^{m-1}}(u) d y_{1} d g d u d h .
\end{gathered}
$$

Here $P^{0}\left(\mathrm{SO}_{2 m}\right)=\mathrm{SO}_{2 m} S_{1}$ is the subgroup of the standard maximal parabolic subgroup of $\mathrm{SO}_{2 m+2}$ whose Levi part is $\mathrm{GL}_{1} \times \mathrm{SO}_{2 m}$. The character $\psi_{Y_{1}}$ is given by $\psi_{Y_{1}}\left(y_{1}\right)=\psi\left(r_{1}\right)$, where we use the coordinates given in (8).

Consider the space of double cosets $P^{0}\left(\mathrm{SO}_{2 m}\right)(F) \backslash \mathrm{SO}_{2 m+2}(F) /$ $\mathrm{SO}_{2 m+1}(F)$. To choose a set of representatives, we first denote the following Weyl elements of $\mathrm{SO}_{4 m}$ by

$$
w_{j}=\operatorname{diag}\left(I_{j-1},\left(\begin{array}{ll} 
& 1 \\
1 &
\end{array}\right), I_{4 m-2 j-2},\left(\begin{array}{ll} 
& 1 \\
1 &
\end{array}\right), I_{j-1}\right) .
$$


Here $1 \leq j \leq 2 m-1$. With this notation, representatives of the double cosets can be chosen as $e$ and $w_{m} w_{m+1} \cdots w_{2 m-2} w_{2 m-1} t$, where $t$ is a certain torus element. We claim that if $m+1>2 r+1$, then the contribution to the integral from the second representative is 0 . Indeed, consider the unipotent subgroup of $\mathrm{SO}_{2 m-1}$ given by all matrices of the form $x(r)=$ $I_{2 m-1}+r\left(e_{1, m}-e_{m, 2 m-1}\right)+r^{*} e_{1,2 m-1}$. Here $r^{*}$ is such that $x(r)$ is a matrix in $\mathrm{SO}_{2 m-1}$. This is a subgroup of $U_{r}^{m-1}$, and assuming that $m+1>2 r+1$, the character $\psi_{U_{r}^{m-1}}$ is trivial on this subgroup. By the parameterization given in (7), we have

$$
(1, x(r))=I_{4 m}+r\left(e_{1,2 m}-e_{1,2 m+1}+e_{2 m, 4 m}-e_{2 m+1,4 m}\right)+r^{*} e_{1,4 m} .
$$

Notice that the group $(1, x(r))$ is a subgroup of $Y_{1}$. Furthermore, after conjugation by $w_{m} w_{m+1} \cdots w_{2 m-2} w_{2 m-1} t$, this group is conjugated to the subgroup of $Y_{1}$ given by all matrices of the form $I_{4 m}+r\left(e_{1, m}-e_{1,3 m+1}+e_{m, 4 m}-\right.$ $\left.e_{3 m+1,4 m}\right)+r^{*} e_{1,4 m}$. It follows from the definition of $\psi_{Y_{1}}$ that this character is not trivial on this group, and after a change of variables in $Y_{1}$, we obtain $\int_{F \backslash \mathbf{A}} \psi(\alpha r) d r$ as inner integration. Here $\alpha \in F^{*}$ is a factor which is obtained from the torus element $t$. Thus, this integral is 0 .

We are left with the representative $e$. Thus, the above integral is equal to

$$
\begin{aligned}
& \iint_{P^{0}\left(\mathrm{SO}_{2 m-1}\right)(F) \backslash \mathrm{SO}_{2 m+1}(\mathbf{A})} \int_{Y_{1}(F) \backslash Y_{1}(\mathbf{A})} \varphi_{\pi}(g) \\
& \quad \times \theta_{\tau, 4 m}\left(y_{1}(g, u h)\right) \psi_{Y_{1}}\left(y_{1}\right) \psi_{U_{r}^{m-1}}(u) d y_{1} d g d u d h .
\end{aligned}
$$

Here $P^{0}\left(\mathrm{SO}_{2 m-1}\right)=\mathrm{SO}_{2 m-1} V_{1}^{m-1}$ is the subgroup of the maximal parabolic subgroup of $\mathrm{SO}_{2 m+1}$ whose Levi part is $\mathrm{GL}_{1} \times \mathrm{SO}_{2 m-1}$. The group $V_{1}^{m-1}$ was defined right after the definition of $\mathcal{P}_{r}(\pi, \tau)$. The other variables are integrated as before.

As subgroups of $\mathrm{SO}_{4 m}$, we have $V_{1}^{m-1} Y_{1}=L_{1}$, where $L_{1}$ was defined right after (8). Also, $V_{1}^{m-1}$ is a subgroup of $U_{r}^{m-1}$. Hence, if we factor the integration over that group, the above integral is equal to

$$
\begin{aligned}
& \iint_{U_{r}^{m-1}(F) V_{1}^{m-1}(\mathbf{A}) \backslash U_{r}^{m-1}(\mathbf{A})} \iint_{L_{1}(F) \backslash L_{1}(\mathbf{A})} \varphi_{\pi}(g) \\
& \quad \times \theta_{\tau, 4 m}(l(g, u h)) \psi_{L_{1}}^{\prime}(l) \psi_{U_{r}^{m-1}}(u) d l d g d u d h .
\end{aligned}
$$

Here, the character $\psi_{L_{1}}^{\prime}$ is defined as follows. For $l=\left(l_{i, j}\right) \in L_{1}$, we have $\psi_{L_{1}}^{\prime}(l)=\psi\left(l_{1,2}+l_{1, m}\right)$. Notice that $V_{1}^{m-1} \backslash U_{r}^{m-1}$ can be identified with $U_{r}^{m-2}$. Denote $z_{1}=w_{2} w_{3} \cdots w_{m-1} x_{\gamma_{1}}(1)$, where $x_{\gamma_{1}}(1)=I_{4 m}+e_{2, m}-$ $e_{3 m+1,4 m-1}$. Then $z_{1} \in \mathrm{SO}_{4 m}(F)$. Hence, $\theta_{\tau, 4 m}(l(g, u h))=\theta_{\tau, 4 m}\left(z_{1} l(g, u h)\right)$. 
Conjugating $z_{1}$ to the right and then changing variables, we obtain

$$
\begin{gathered}
\iint_{P^{0}\left(\mathrm{SO}_{2 m-1}\right)(F) \backslash \mathrm{SO}_{2 m+1}(\mathbf{A})} \int_{U_{r}^{m-2}(F) \backslash U_{r}^{m-2}(\mathbf{A})} \varphi_{\pi}(g) \\
\times \theta_{\tau, 4 m}^{L_{1}, \psi}\left(z_{1}(g, u h)\right) \psi_{U_{r}^{m-2}}(u) d u d g d h,
\end{gathered}
$$

where $h$ is integrated over $\operatorname{Sp}_{2 r}(F) \backslash \operatorname{Sp}_{2 r}(\mathbf{A})$. Also, we denote

$$
\theta_{\tau, 4 m}^{L_{1}, \psi}\left(z_{1}(g, u h)\right)=\int_{L_{1}(F) \backslash L_{1}(\mathbf{A})} \theta_{\tau, 4 m}\left(l z_{1}(g, u h)\right) \psi_{L_{1}}(l) d l,
$$

where $\psi_{L_{1}}$ is defined as follows. For $l=\left(l_{i, j}\right) \in L_{1}$, we define $\psi_{L_{1}}(l)=\psi\left(l_{1,2}\right)$. In fact, we have

$$
\theta_{\tau, 4 m}^{L_{1}, \psi}\left(z_{1}(g, u h)\right)=\theta_{\tau, 4 m}^{L_{2}, \psi}\left(z_{1}(g, u h)\right)
$$

where the character $\psi_{L_{1}}$ is extended trivially from $L_{1}$ to $L_{2}$. Indeed, to derive this identity, we expand integral (10) along the group $L_{1} \backslash L_{2}$ (more precisely, the A-rational points of $L_{1} \backslash L_{2}$ modulo the $F$-rational points of $\left.L_{1} \backslash L_{2}\right)$. The group $\mathrm{SO}_{4 m-4}(F)$ acts on this expansion with three types of orbits. In a similar way as in the expansion along $Y_{1}$ and then $Y_{2}$, as was done right after (8), we show that only the constant term gives a nonzero contribution. Thus, the above identity holds.

In integral (9), we conjugate the matrices $(1, u)$, where $u \in U_{r}^{m-2}$, across $z_{1}$, and we obtain the matrices $(1, u)_{1}$ given by

$$
u \mapsto(1, u)_{1}=\left(\begin{array}{lll}
I_{2} & & \\
& (1, u) & \\
& & I_{2}
\end{array}\right) .
$$

Here we view $(1, u)$ as an element in $\mathrm{SO}_{4 m-4}$ embedded in $\mathrm{SO}_{4 m}$ as above that is parameterized as in (7). In this way, we see that we reduced the computations to the case with $m-1$ instead of $m$.

We repeat this process $m-2 r-2$ times. We deduce that integral (6) is not 0 for some choice of data if and only if the integral

$$
\begin{gathered}
\iint_{P^{0}\left(\mathrm{SO}_{4 r+5}\right)(F) \backslash \mathrm{SO}_{2 m+1}(\mathbf{A})} \int_{U_{r}^{2 r+1}(F) \backslash U_{r}^{2 r+1}(\mathbf{A})} \varphi_{\pi}(g) \\
\times \theta_{\tau, 4 m}^{L_{2(m-2 r-2)}, \psi}(\widetilde{z}(g, u h)) \psi_{U_{r}^{2 r+1}}(u) d u d g d h
\end{gathered}
$$


is not 0 for some choice of data. Here $h$ is integrated as in integral (9), and $\widetilde{z}$ is defined as follows. For $1 \leq i \leq m-2 r-2$, let $x_{\gamma_{i}}(1)=I_{4 m}+$ $e_{2 i, m+i-1}-e_{3 m-i+2,4 m-2 i+1}$. Define $z_{i}=w_{2 i} w_{2 i+1} \cdots w_{m+i-2} x_{\gamma_{i}}(1)$. Then $\widetilde{z}=z_{m-2 r-2} \cdots z_{2} z_{1}$. Finally, we let $\theta_{\tau, 4 m}^{L_{2(m-2 r-2)}, \psi}$ be defined as in (10) where we integrate over $L_{2(m-2 r-2)}(F) \backslash L_{2(m-2 r-2)}(\mathbf{A})$, and $\psi_{L_{2(m-2 r-2)}}(l)$ is defined as follows. For $l=\left(l_{i, j}\right) \in L_{2(m-2 r-2)}$, we define

$$
\psi_{L_{2(m-2 r-2)}}(l)=\psi\left(l_{1,2}+l_{3,4}+l_{5,6}+\cdots+l_{2(m-2 r-2)-1,2(m-2 r-2)}\right) .
$$

Next we expand integral (11) along the unipotent group $Y_{2(m-2 r-2)+1}$ defined as the group of all matrices of the form

$$
\begin{aligned}
y= & I_{4 m}+\sum_{i=1}^{4 r+6}\left[r_{i}\left(e_{2 m-4 r-3,2 m-2 r+i-3}-e_{2 m+2 r-i+4,2 m+4 r+4}\right)\right] \\
& +r^{*} e_{2 m-4 r-3,2 m+4 r+4} .
\end{aligned}
$$

Here $r^{*}$ is defined in a similar way as in (8). The group $\mathrm{SO}_{4 r+6}(F)$, embedded as $g \mapsto \operatorname{diag}\left(I_{2 m-2 r-3}, g, I_{2 m-2 r-3}\right)$, acts on this expansion with three types of orbits. One type of orbit is the constant term, the second type corresponds to nonzero vectors with 0 length, and the third corresponds to all vectors which have nonzero length. As in the case of the expansion of $Y_{1}$, we show that the contributions to the integral from the constant term and from the nonzero length vectors are 0 . We are left with the orbit of all nonzero vectors which have 0 length. Thus, integral (11) is equal to

$$
\begin{aligned}
& \sum_{\gamma \in P^{0}\left(\mathrm{SO}_{4 r+4}\right)(F) \backslash \mathrm{SO}_{4 r+6}(F)} \int_{Y_{2(m-2 r-2)+1}(F) \backslash Y_{2(m-2 r-2)+1}(\mathbf{A})} \varphi_{\pi}(g) \\
& \times \theta_{\tau, 4 m}^{L_{2(m-2 r-2)}, \psi}(y \widetilde{z} \gamma(g, u h)) \psi_{Y_{2(m-2 r-2)+1}}(y) \psi_{U_{r}^{2 r+1}}(u) d y d u d g d h .
\end{aligned}
$$

Here $\psi_{Y_{2(m-2 r-2)+1}}$ is defined as follows. In the coordinates of this group, as given in $(12)$, we have $\psi_{Y_{2(m-2 r-2)+1}}(y)=\psi\left(r_{1}\right)$. All other variables are integrated as in (11).

Next we consider the space of double cosets $P^{0}\left(\mathrm{SO}_{4 r+4}\right)(F) \backslash \mathrm{SO}_{4 r+6}(F) /$ $\mathrm{SO}_{4 r+5}(F)$. The space contains two types of elements, and as representatives we may choose $e$ and the elements $w_{2(m-r-1)} w_{2 m-2 r-1} \cdots w_{2 m-2} w_{2 m-1} h(\zeta)$. Here $h(\zeta)=\operatorname{diag}\left(I_{2 m-1}, \zeta, \zeta^{-1}, I_{2 m-1}\right)$, where $\zeta \in F^{*}$. The contribution to the above integral from the identity element is 0 . This follows from the 
definition of the character $\psi_{U_{r}^{2 r+1}}$. As for the other representatives, it also follows from the definition of $\psi_{U_{r}^{2 r+1}}$ that if $\zeta \neq 1$, then the contribution to the above integral is 0 . Indeed, conjugating the element $u \in U_{r}^{2 r+1}$ across $w_{2(m-r-1)} w_{2 m-2 r-1} \cdots w_{2 m-2} w_{2 m-1} h(\zeta)$ and then changing variables in $Y_{2(m-2 r-2)+1}$, we obtain the integral $\int \psi((1-\zeta) r) d r$ as inner integration. Here we integrate over $F \backslash \mathbf{A}$, and hence this integral is 0 unless $\zeta=1$. Thus, we are left with the Weyl element $w_{0}=w_{2(m-r-1)} w_{2 m-2 r-1} \cdots w_{2 m-2} w_{2 m-1}$. The stabilizer of $w_{0}$ inside $\mathrm{SO}_{4 r+5}$ is $\mathrm{SO}_{4 r+4}$, and hence we may collapse summation with integration in the above integral. It follows that $g$ is now integrated over $V_{m-2 r-2}^{m}(F) \mathrm{SO}_{4(r+1)}(F) \backslash \mathrm{SO}_{2 m+1}(\mathbf{A})$.

We have the group identity

$$
L_{2(m-2 r-2)+1}=L_{2(m-2 r-2)} Y_{2(m-2 r-2)+1} w_{0} U_{r}^{2 r+1} w_{0}^{-1} .
$$

After a suitable conjugation, the above integral is equal to

$$
\iint_{U_{r}^{2 r}(F) \backslash U_{r}^{2 r}(\mathbf{A})} \varphi_{\pi}(g) \theta_{\tau, 4 m}^{L_{2(m-2 r-2)+1}, \psi}\left(z_{0}(g, u h)\right) \psi_{U_{r}^{2 r}}(u) d u d g d h .
$$

Here $h$ is integrated over $\operatorname{Sp}_{2 r}(F) \backslash \mathrm{Sp}_{2 r}(\mathbf{A})$, and $g$ is integrated over the quotient given by $V_{m-2 r-2}^{m}(F) \mathrm{SO}_{4(r+1)}(F) \backslash \mathrm{SO}_{2 m+1}(\mathbf{A})$. The element $z_{0} \in$ $\mathrm{SO}_{4 m}(F)$ is equal to $z^{\prime} \widetilde{z} w_{0}$, where $z^{\prime}=w_{2 m-4 r-3} w_{2 m-4 r-2} \cdots w_{2 m-2 r-3}$. Finally, $\theta_{\tau, 4 m}^{L_{2(m-2 r-2)+1}, \psi}$ is defined in a similar way as in (10), where the character of the group $L_{2(m-2 r-2)+1}$ is defined as follows. If $l=\left(l_{i, j}\right)$, then the character is defined by $\psi\left(l_{1,2}+l_{3,4}+l_{5,6}+\cdots+l_{2(m-2 r-2)-1,2(m-2 r-2)}+\right.$ $\left.l_{2(m-2 r-2)+1,2(m-2 r-2)+2}\right)$. Also, in a similar way as is shown right after $(10)$, we have $\theta_{\tau, 4 m}^{L_{2(m-2 r-2)+1}, \psi}=\theta_{\tau, 4 m}^{L_{2(m-2 r-2)+2}, \psi}$, where the character is extended trivially.

Next we factor the integration $V_{m-2 r-2}^{m}(F) \mathrm{SO}_{4(r+1)}(F) \backslash \mathrm{SO}_{2 m+1}(\mathbf{A})$ into

$$
\begin{gathered}
\int_{V_{m-2 r-2}^{m}(\mathbf{A}) \mathrm{SO}_{4(r+1)}(\mathbf{A}) \backslash \mathrm{SO}_{2 m+1}(\mathbf{A})} \int_{\mathrm{SO}_{4(r+1)}(F) \backslash \mathrm{SO}_{4(r+1)}(\mathbf{A})} \\
\quad \times \int_{V_{m-2 r-2}^{m}(F) \backslash V_{m-2 r-2}^{m}(\mathbf{A})} \cdot
\end{gathered}
$$

A matrix multiplication and a change of variables imply that, for all $v \in$ $V_{m-2 r-2}^{m}(\mathbf{A})$, we have

$$
\theta_{\tau, 4 m}^{L_{2(m-2 r-2)+2}, \psi}\left(z_{0}(v g, u h)\right)=\psi_{V_{m-2 r-2}^{m}}(v) \theta_{\tau, 4 m}^{L_{2(m-2 r-2)+2}, \psi}\left(z_{0}(g, u h)\right) .
$$


We denote

$$
\varphi_{\pi}^{V, \psi}(g)=\int_{V_{m-2 r-2}^{m}(F) \backslash V_{m-2 r-2}^{m}(\mathbf{A})} \varphi_{\pi}(v g) \psi_{V_{m-2 r-2}^{m}}(v) d v .
$$

Using the same notation and conjugating by $z_{0}$, integral (13) is equal to

$$
\begin{aligned}
& \iint_{U_{r}^{2 r, z_{0}}(F) \backslash U_{r}^{2 r, z_{0}}(\mathbf{A})} \varphi_{\pi}^{V, \psi}\left(g_{0} g\right) \\
& \quad \times \theta_{\tau, 4 m}^{L_{2(m-2 r-1)}, \psi}\left(\widetilde{y}\left(g_{0}, h\right)_{1} z_{0}(g, 1)\right) \widetilde{\psi}(y) d y d g_{0} d g d h .
\end{aligned}
$$

Here

$$
\widetilde{y}\left(g_{0}, h\right)_{1}=\left(\begin{array}{cccccc}
I_{a} & & & & \\
& I_{2 r} & & y & \\
& & I_{4(r+1)} & & \\
& & & I_{2 r} & \\
& & & & I_{a}
\end{array}\right)\left(\begin{array}{ccccc}
I_{a} & & & & \\
& h & & & \\
& & g_{0} & & \\
& & h^{*} & \\
& & & I_{a}
\end{array}\right),
$$

where $a=2(m-2 r-1)$ and $g_{0} \in \mathrm{SO}_{4(r+1)}$. In the above integral, $g_{0}$ is integrated over $\mathrm{SO}_{4(r+1)}(F) \backslash \mathrm{SO}_{4(r+1)}(\mathbf{A})$, the variable $g$ is integrated over $V_{m-2 r-2}^{m}(\mathbf{A}) \mathrm{SO}_{4(r+1)}(\mathbf{A}) \backslash \mathrm{SO}_{2 m+1}(\mathbf{A})$, and all other variables are integrated as before. We also denote $U_{r}^{2 r, z_{0}}=z_{0} U_{r}^{2 r} z_{0}^{-1}$, and we define the character $\widetilde{\psi}$ by $\widetilde{\psi}(y)=\psi\left(y_{1,1}+y_{2,2}+\cdots+y_{r, r}\right)$.

Consider the group $\mathrm{SO}_{8 r+4}$, embedded inside $\mathrm{SO}_{4 m}$, as $t \mapsto \operatorname{diag}\left(I_{a}, t, I_{a}\right)$, where $t \in \mathrm{SO}_{8 r+4}$. It follows from the above that the matrices $\widetilde{y}\left(g_{0}, h\right)_{1}$ are embedded inside the maximal parabolic subgroup of $\mathrm{SO}_{8 r+4}$ whose Levi part is $\mathrm{GL}_{2 r} \times \mathrm{SO}_{4 r+4}$. Then, the unipotent radical of this parabolic group, which we will denote by $W_{r}$, has a structure of a generalized Heisenberg group. Its center is the group $U_{r}^{2 r, z_{0}}$. To proceed with the computations, we now apply the theory of Fourier-Jacobi coefficients, as developed in [Ik]. In our context, this theory asserts that as a function of $g_{0}$ and $h$, the space of functions

$$
\begin{aligned}
& \theta_{\phi, \psi}^{\mathrm{Sp}_{8 r(r+1)}}\left(\left(g_{0}, h\right)\right) \int_{W_{r}(F) \backslash W_{r}(\mathbf{A})} \theta_{\tau, 4 m}^{L_{2(m-2 r-1)}, \psi}\left(w\left(g_{0}, h\right)_{1} z_{0}(g, 1)\right) \\
& \quad \times \theta_{\phi_{1}, \psi}^{\mathrm{Sp}_{8 r(r+1)}}\left(l(w)\left(g_{0}, h\right)\right) d w
\end{aligned}
$$

is a dense subspace inside the space of functions

$$
\left(g_{0}, h\right) \mapsto \int_{U_{r}^{2 r, z_{0}}(F) \backslash U_{r}^{2 r, z_{0}}(\mathbf{A})} \theta_{\tau, 4 m}^{L_{2(m-2 r-1)}, \psi}\left(\widetilde{y}\left(g_{0}, h\right)_{1} z_{0}(g, 1)\right) \widetilde{\psi}(y) d y .
$$


In (15), the function $\theta_{\phi, \psi}^{\mathrm{Sp}_{8 r(r+1)}}$ is the $\theta$-function defined on the double cover of $\operatorname{Sp}_{8 r(r+1)}(\mathbf{A})$. Also, $\phi$ and $\phi_{1}$ are suitable Schwartz functions in $\mathcal{S}\left(\mathbf{A}^{2 r \times 2(r+1)}\right)$. We denote by $l$ the projection from $W_{r}$ onto the Heisenberg group with $8 r(r+1)+1$ variables.

Denote the integral in $(15)$ by $F\left(g_{0}, h ; g\right)$. Here, $g$ is a fixed element in $\mathrm{SO}_{2 m+1}(\mathbf{A})$. We have the following.

Lemma 1. For all $h \in \mathrm{Sp}_{2 r}(\mathbf{A})$, we have $F\left(g_{0}, h ; g\right)=F\left(g_{0}, 1 ; g\right)$.

The proof of this lemma will be given after the proof of the theorem. Assuming the lemma, we deduce that integral (14) is not 0 for some choice of data if and only if the integral

$$
\int \varphi_{\pi}^{V, \psi}\left(g_{0} g\right) \theta_{\phi, \psi}^{\mathrm{Sp}_{8 r(r+1)}}\left(\left(g_{0}, h\right)\right) F\left(g_{0}, 1 ; g\right) d g_{0} d g d h
$$

is not 0 for some choice of data. Here all variables are integrated as before.

Next we calculate the inner integration of (16) along the variable $h$, which is

$$
\int_{\operatorname{Sp}_{2 r}(F) \backslash \operatorname{Sp}_{2 r}(\mathbf{A})} \theta_{\phi, \psi}^{\mathrm{Sp}_{8 r(r+1)}}\left(\left(g_{0}, h\right)\right) d h .
$$

This integral may not converge for a general function $\phi \in \mathcal{S}\left(\mathbf{A}^{2 r \times 2(r+1)}\right)$. Thus, we use regularization by a Hecke algebra element at one unramified local place to extend the integral to general Schwartz functions $\phi \in$ $\mathcal{S}\left(\mathbf{A}^{2 r \times 2(r+1)}\right)$. This idea of regularization was used by Ichino [Ich], which extends that of Kudla and Rallis $[\mathrm{KR}]$ on a regularized Siegel-Weil formula. The current case was done in [JS, Theorem 2.4]. The theorem states that there exists a Hecke algebra element $\alpha_{\nu_{0}}$ in the Hecke algebra of $\mathrm{SO}_{4(r+1)}$ at a finite local place $\nu_{0}$ such that the integral

$$
c_{\alpha_{\nu_{0}}}^{-1} \cdot \int_{\operatorname{Sp}_{2 r}(F) \backslash \operatorname{Sp}_{2 r}(\mathbf{A})} \theta_{\omega_{\psi_{\nu_{0}}}\left(1, \alpha_{\nu_{0}}\right) \phi, \psi}^{\mathrm{Sp}_{8 r}(r+1)}\left(\left(g_{0}, h\right)\right) d h
$$

converges absolutely and is the unique extension of integral (17) to general Schwartz functions $\phi$ in $\mathcal{S}\left(\mathbf{A}^{2 r \times 2(r+1)}\right)$. The second part of [JS, Theorem 2.4] states that the integral (17) or, equivalently, (18) is identified as the residue at $s=(r+1) /(2 r+1)$ of the Eisenstein series $E(g, s)$ on $\mathrm{SO}_{4(r+1)}(\mathbf{A})$, which is denoted by $\Theta_{\left[2^{2 r} 1^{4}\right]}$. 
Hence, integral (16) is not 0 for some choice of data if and only if the integral

$$
\begin{aligned}
& \int_{V_{m-2 r-2}^{m}(\mathbf{A}) \mathrm{SO}_{4(r+1)}(\mathbf{A}) \backslash \mathrm{SO}_{2 m+1}(\mathbf{A})} \int_{\mathrm{SO}_{4(r+1)}(F) \backslash \mathrm{SO}_{4(r+1)}(\mathbf{A})} \varphi_{\pi}^{V, \psi}\left(g_{0} g\right) \\
& \quad \times F\left(g_{0}, 1 ; g\right) \theta_{\left[2^{2 r} 1^{4}\right]}\left(g_{0}\right) d g_{0} d g
\end{aligned}
$$

is not 0 for some choice of data. By applying a standard argument, for example, as in [GS, Section 7], we deduce that the above integral is nonzero for some choice of data if and only if the integral

$$
\int_{\mathrm{SO}_{4(r+1)}(F) \backslash \mathrm{SO}_{4(r+1)}(\mathbf{A})} \varphi_{\pi}^{V, \psi}\left(g_{0}\right) F\left(g_{0}, 1 ; 1\right) \theta_{\left[2^{2 r} 1^{4}\right]}\left(g_{0}\right) d g_{0}
$$

is not 0 for some choice of data. Applying Proposition 2 from the appendix, it follows from the definitions of the group $L_{2(m-2 r-1)}$ and the character defined on this group that the above integral is not 0 for some choice of data if and only if the integral

$$
\begin{aligned}
& \int \varphi_{\pi}^{V, \psi}\left(g_{0}\right) \int_{W_{r}(F) \backslash W_{r}(\mathbf{A})} \theta_{\tau, 8 r+4}\left(w g_{0}\right) \\
& \quad \times \theta_{\phi_{1}, \psi}^{\mathrm{Sp}_{8 r(r+1)}}\left(l(w)\left(g_{0}, 1\right)\right) d w \theta_{\left[2^{2 r} 1^{4}\right]}\left(g_{0}\right) d g_{0}
\end{aligned}
$$

is not 0 for some choice of data. Here $g_{0}$ is integrated as before. Unfolding the $\theta$-function (for the action of the Weil representation; see [Ik]) and collapsing summation with integration, the inner integral is equal to

$$
\begin{aligned}
\int_{W_{r}^{\prime}(\mathbf{A})} & \int_{X(F) \backslash X(\mathbf{A})} \int_{Y(F) \backslash Y(\mathbf{A})} \theta_{\tau, 8 r+4}\left((x, y) g_{0} w^{\prime}\right) \\
& \times \omega_{\psi}\left(l(x, y) g_{0} l\left(w^{\prime}\right)\right) \phi_{1}(0) d x d y d w^{\prime} \\
= & \int_{W_{r}^{\prime}(\mathbf{A})} \int_{X(F) \backslash X(\mathbf{A})} \int_{Y(F) \backslash Y(\mathbf{A})} \theta_{\tau, 8 r+4}\left((x, y) g_{0} w^{\prime}\right) \\
& \times \psi_{r}(y) \phi_{1}\left(w^{\prime}\right) d x d y d w^{\prime} .
\end{aligned}
$$

Here, the embedding of $X$ and $Y$ in $\mathrm{SO}_{8 r+4}$ is given by

$$
(x, y) g_{0} \mapsto\left(\begin{array}{ccccc}
I_{r} & & x & y_{1} & y_{2} \\
& I_{r} & & y_{3} & y_{1}^{*} \\
& & I_{4 r+4} & & x^{*} \\
& & & I_{r} & \\
& & & & I_{r}
\end{array}\right)\left(\begin{array}{ccc}
I_{2 r} & & \\
& g_{0} & \\
& & I_{2 r}
\end{array}\right), \quad y=\left(\begin{array}{ll}
y_{1} & y_{2} \\
y_{3} & y_{1}^{*}
\end{array}\right) .
$$


The character $\psi_{r}$ is defined by $\psi_{r}(y)=\psi\left(\operatorname{tr} y_{1}\right)$. Since $\phi_{1}$ is an arbitrary Schwartz function, it follows that integral (19) is nonzero for some choice of data if and only if the integral

$$
\begin{aligned}
& \int_{\mathrm{SO}_{4(r+1)}(F) \backslash \mathrm{SO}_{4(r+1)}(\mathbf{A})} \varphi_{\pi}^{V, \psi}\left(g_{0}\right) \int_{X(F) \backslash X(\mathbf{A})} \int_{Y(F) \backslash Y(\mathbf{A})} \theta_{\tau, 8 r+4}\left((x, y) g_{0}\right) \\
& \times \psi_{r}(y) \theta_{\left[2^{2 r} 1^{4}\right]}\left(g_{0}\right) d x d y d g_{0}
\end{aligned}
$$

is not 0 for some choice of data. Next we perform the following series of Fourier expansions. Recall that for the above matrix $y$ to be in the orthogonal group, we have

$$
y=\left(\begin{array}{ll}
y_{1} & y_{2} \\
y_{3} & y_{1}^{*}
\end{array}\right) \in \operatorname{Mat}_{2 r}^{0}=\left\{A \in \operatorname{Mat}_{2 r}: J_{2 r} A+A^{t} J_{2 r}=0\right\},
$$

where $J_{2 r}$ is the matrix of size $2 r$ which has ones on the other diagonal and zeros elsewhere. Let $Y^{0}$ denote the subgroup of $Y$ which consists of all matrices $y$ as above such that $y_{i, j}=0$ for all $i \leq j$. Let $Z_{2 r}^{e}$ denote the group of all matrices of the form

$$
Z_{2 r}=\left\{z=\left(\begin{array}{cc}
z_{1} & z_{2} \\
& I_{r}
\end{array}\right): z_{1} \in Z_{2 r}^{\prime}, z_{2} \in Z_{2 r}^{\prime \prime}\right\}
$$

Here $Z_{2 r}^{\prime}$ consists of all upper unipotent matrices in $\mathrm{GL}_{r}$, and $Z_{2 r}^{\prime \prime}$ consists of all matrices of size $r$ such that $J_{r} z_{2}$ is lower triangular. Also, the matrix $J_{r}$ is defined in a similar way as we defined $J_{2 r}$. We will denote by $Z_{2 r}$ the subgroup of $Z_{2 r}^{e}$ which consists of all matrices above such that the diagonal entries of $J_{r} z_{2}$ are also 0 .

We embed these groups in $\mathrm{SO}_{8 r+4}$ as all matrices of the form $z \mapsto$ $\operatorname{diag}\left(z, I_{4 r+4}, z^{*}\right)$, and we continue to denote it by $Z_{2 r}^{e}$ or $Z_{2 r}$.

In integral (21) we expand the function $\theta_{\tau, 8 r+4}\left((x, y) g_{0}\right)$ along the group $Z_{2 r}$. Collapsing summation and integration over $Y^{0}$, integral $(21)$ is equal to

$$
\begin{aligned}
& \iint_{Y^{0}(\mathbf{A})} \varphi_{\pi}^{V, \psi}\left(g_{0}\right) \iint_{Z_{2 r}(F) \backslash Z_{2 r}(\mathbf{A})} \theta_{\tau, 8 r+4}\left(z\left(x, y y_{0}\right) g_{0}\right) \\
& \quad \times \psi_{r}(y) \theta_{\left[2^{2 r} 1^{4}\right]}\left(g_{0}\right) d z d x d y d y_{0} d g_{0} .
\end{aligned}
$$

Here $g_{0}$ and $x$ are integrated as before, $y$ is integrated over $Y(F) Y^{0}(\mathbf{A}) \backslash$ $Y(\mathbf{A})$, and $y_{0}$ is integrated over $Y^{0}(\mathbf{A})$. 
In fact, we can extend trivially the integration from $Z_{2 r}$ to $Z_{2 r}^{e}$. Indeed, we identify the quotient $Z_{2 r} \backslash Z_{2 r}^{e}$ with the group of unipotent matrices $I_{8 r+4}+$ $\sum_{i=1}^{r} r_{i}\left(e_{i, 2 r-i+1}-e_{6 r+i+4,8 r-i+5}\right)$. Thus, expanding integral (22) along this quotient, we get two terms. The first term corresponds to the constant term, and the second term corresponds to all other elements in the expansion. The second term contributes 0 to the expansion. Indeed, a similar argument as follows after (8) shows that this term will contain as inner integration a Fourier coefficient of $\theta_{\tau, 8 r+4}$ which corresponds to the unipotent orbit with partition $\left(31^{8 r+1}\right)$. It follows from Proposition 1 in the appendix, applied to $8 r+4$ instead of to $4 m$, that this Fourier coefficient is 0 . Thus, we can replace in integral (22) the integration over $Z_{2 r}(F) \backslash Z_{2 r}(\mathbf{A})$ by the integration over $Z_{2 r}^{e}(F) \backslash Z_{2 r}^{e}(\mathbf{A})$.

Next we define the following Weyl element $\widetilde{w}$ of $\mathrm{SO}_{8 r+4}$. For all $1 \leq i \leq r$, set

$$
\widetilde{w}_{2 i-1, i}=\widetilde{w}_{2 i, 6 r+i+4}=\widetilde{w}_{8 r-i+5,8 r-2 i+6}=\widetilde{w}_{2 r-i+1,8 r-2 i+5}=1 .
$$

Also, for $2 r+1 \leq i \leq 6 r+4$, set $\widetilde{w}_{i, i}=1$. All other entries of $\widetilde{w}$ are 0 . Matrix multiplication implies that $L_{2 r}^{\prime}=\widetilde{w}\left(Z_{2 r}^{e} \cdot X \cdot Y^{0} \backslash Y\right) \widetilde{w}^{-1}$ is a subgroup of $L_{2 r}$. The groups $L_{i}$ were defined as subgroups of $\mathrm{SO}_{4 m}$ right after (8). In a similar way, we define them as subgroups of any even orthogonal group, in particular, for $\mathrm{SO}_{8 r+4}$. Similarly, we define the character $\psi_{L_{2 r}}(l)$. More precisely, for $l=\left(l_{i, j}\right) \in L_{2 r}$ we define $\psi_{L_{2 r}}(l)=\psi\left(l_{1,2}+l_{3,4}+\cdots+l_{2 r-1,2 r}\right)$. It follows from the matrix multiplication that, after the conjugation by $\widetilde{w}$, the character $\psi_{r}$ is conjugated to $\psi_{L_{2 r}}$ restricted to $L_{2 r}^{\prime}$. Thus, integral (22) is not 0 for some choice of data if and only if the integral

$$
\begin{aligned}
& \int_{\mathrm{SO}_{4(r+1)}(F) \backslash \mathrm{SO}_{4(r+1)}(\mathbf{A})} \int_{Y^{0}(\mathbf{A})} \varphi_{\pi}^{V, \psi}\left(g_{0}\right) \\
& \quad \times \theta_{\tau, 8 r+4}^{L_{2 r}^{\prime}, \psi}\left(\widetilde{w} y_{0} g_{0}\right) \theta_{\left[2^{2 r} 1^{4}\right]}\left(g_{0}\right) d y_{0} d g_{0}
\end{aligned}
$$

is not 0 for some choice of data. Here $\theta_{\tau, 8 r+4}^{L_{2 r}^{\prime}, \psi}$ is defined in a similar way as in (10). We claim that $\theta_{\tau, 8 r+4}^{L_{2 r}^{\prime}, \psi}=\theta_{\tau, 8 r+4}^{L_{2 r}, \psi}$. In other words, we claim that integral (23) is not 0 for some choice of data if and only if the integral

$$
\begin{aligned}
& \int_{\mathrm{SO}_{4(r+1)}(F) \backslash \mathrm{SO}_{4(r+1)}(\mathbf{A})} \int_{Y^{0}(\mathbf{A})} \varphi_{\pi}^{V, \psi}\left(g_{0}\right) \\
& \times \theta_{\tau, 8 r+4}^{L_{2 r}, \psi}\left(\widetilde{w} y_{0} g_{0}\right) \theta_{\left[2^{2 r} 1^{4}\right]}\left(g_{0}\right) d y_{0} d g_{0}
\end{aligned}
$$


is not 0 for some choice of data. This argument follows in a similar way as the argument given right after (10) when we extended the integration from $L_{1}$ to $L_{2}$ as defined there. In our case, we expand integral (23) along the quotient $L_{2 r}^{\prime} \backslash L_{2 r}$. Each element in this expansion which is not the constant term contributes 0 . This follows again by using Proposition 1 from the appendix. Indeed, all Fourier coefficients which are not the constant term correspond to unipotent orbits which are larger or not related to the partition $\left(2^{2(r+1)}\right)$.

Arguing as above, in a similar way as in [GS], we deduce that integral (24) is not 0 for some choice of data if and only if the integral

$$
\int_{\mathrm{SO}_{4(r+1)}(F) \backslash \mathrm{SO}_{4(r+1)}(\mathbf{A})} \varphi_{\pi}^{V, \psi}\left(g_{0}\right) \theta_{\tau, 8 r+4}^{L_{2 r}, \psi}\left(\widetilde{w} g_{0}\right) \theta_{\left[2^{2 r} 1^{4}\right]}\left(g_{0}\right) d g_{0}
$$

is not 0 for some choice of data. From the definition of $\widetilde{w}$, we have $\widetilde{w} g_{0}=$ $g_{0} \widetilde{w}$. Applying Proposition 2 from the appendix, we deduce that the above integral is not 0 for some choice of data if and only if the integral

$$
\int_{\mathrm{SO}_{4(r+1)}(F) \backslash \mathrm{SO}_{4(r+1)}(\mathbf{A})} \varphi_{\pi}^{V, \psi}\left(g_{0}\right) \theta_{\tau, 4 r+4}\left(g_{0}\right) \theta_{\left[2^{2 r} 1^{4}\right]}\left(g_{0}\right) d g_{0}
$$

is not 0 for some choice of data. But this is integral $\mathcal{P}_{r}(\pi, \tau)$.

To complete the proof of this step, we still need to prove Lemma 1, which is done in Section 3.2.

Next we need to prove the converse of Conjecture 2. In other words, we need to prove that if $\mathcal{P}_{r}(\pi, \tau)$ is not 0 for some choice of data, then $\pi$ is an endoscopic representation with respect to $\mathrm{SO}_{3} \times \mathrm{SO}_{2 r+1} \times \mathrm{SO}_{2(m-r)-1}$. This is done by reversing the arguments in the first part. Indeed, starting with the fact that $\mathcal{P}_{r}(\pi, \tau)$ is not 0 for some choice of data, we obtain by applying the converse of the above computations that the integral (6) is not 0 for some choice of data. But this implies that if we denote by $\sigma$ the space of the representation of $\mathrm{SO}_{2 m-1}(\mathbf{A})$, generated by the functions given by integral (26) in the appendix, then $\sigma$ is not 0 , and by (5), we obtain that $\mathcal{Q}_{r}(\sigma)$ is not 0 . Also, from Theorem 4 in the appendix, we deduce that $\sigma$ is a cuspidal representation. Moreover, since $\sigma$ is not 0 , then integral (27) in the appendix is not 0 for some choice of data, which implies that $\pi$ is endoscopic with respect to $\tau$ and $\sigma$. Applying Conjecture 1, we obtain that $\sigma$ is endoscopic with respect to $\mathrm{SO}_{2 r+1} \times \mathrm{SO}_{2(m-r)-1}$, and we are done.

This completes the proof. 


\subsection{Proof of Lemma 1}

Let $p(k)=I_{2 r}+k e_{1,2 r}$. Then the group generated by these matrices is a unipotent subgroup of $\mathrm{Sp}_{2 r}$ which corresponds to the highest root of $\mathrm{Sp}_{2 r}$. Thus, to prove the lemma, it is enough to prove that $F\left(g_{0}, p(k) h ; g\right)=$ $F\left(g_{0}, h ; g\right)$ for all $k \in \mathbf{A}$. To prove that, it is enough to prove that the integral

$$
\int_{F \backslash \mathbf{A}} F\left(g_{0}, p(k) h ; g\right) \psi(\alpha k) d k
$$

is 0 for all choice of data and all $\alpha \in F^{*}$. To prove that, we may assume that $g_{0}, h$, and $g$ are the identity elements. Thus, from the definition of the function $F\left(g_{0}, h ; g\right)$, we need to prove that the integral

$$
\int_{W_{r}(F) \backslash W_{r}(\mathbf{A})} \int_{F \backslash \mathbf{A}} \theta_{\tau, 8 r+4}(w p(k)) \theta_{\phi_{1}, \psi}^{\mathrm{Sp}_{8 r(r+1)}}(l(w)(1, p(k))) \psi(\alpha k) d k d w
$$

is 0 for all choice of data. We unfold the $\theta$-function, and as in (20) and (21), we deduce that it is enough to prove that the integral

$$
\int_{X(F) \backslash X(\mathbf{A})} \int_{Y(F) \backslash Y(\mathbf{A})} \int_{F \backslash \mathbf{A}} \theta_{\tau, 8 r+4}((x, y) p(k)) \psi_{r}(y) \psi(\alpha k) d k d x d y
$$

is 0 for all choice of data.

Defining the groups $Z_{2 r}$ and $Z_{2 r}^{e}$ as defined right before (22), it is enough to prove that the integral

$$
\begin{aligned}
& \int_{Y^{0}(\mathbf{A})} \int_{X(F) \backslash X(\mathbf{A})} \int_{Y(F) \backslash Y(\mathbf{A})} \int_{F \backslash \mathbf{A}} \int_{Z_{2 r}^{e}(F) \backslash Z_{2 r}^{e}(\mathbf{A})} \theta_{\tau, 8 r+4}\left(z\left(x, y y_{0}\right) p(k)\right) \\
& \times \psi_{r}(y) \psi(\alpha k) d z d k d x d y d y_{0}
\end{aligned}
$$

is 0 for all choice of data. The subgroup generated by all $p(k)$ is contained in $Z_{2 r} \backslash Z_{2 r}^{e}$, and as explained right after (22), the character $\psi_{r}$ is trivial on this quotient. Hence, it is trivial on $p(k)$, and conjugating this matrix to the left and changing variables, we obtain the integral $\int_{F \backslash \mathbf{A}} \psi(\alpha k) d k$ as inner integration. Since $\alpha \in F^{*}$, this integral is 0 . Hence, integral (25) is 0 for all choice of data.

Hence, this completes the proof.

\section{Appendix}

In this appendix, we review the basic results regarding the construction of the endoscopic lifting as it appears in [G3]. Some more details can also 
be found in [G1]. Since we need the details only for a special case, we will concentrate only on the relevant details.

Let $\tau$ denote an irreducible cuspidal representation of $\mathrm{GL}_{2}$ with a trivial central character. Let $\Theta_{\tau, 4 m}$ denote the residue representation defined on $\mathrm{SO}_{4 m}(\mathbf{A})$ as constructed in [G3, p. 461, Case 5]. (In the notation of that reference, take $m=1$ and $n=r$.) The basic property of this representation is given by the following.

Proposition 1. We have $\mathcal{O}_{\mathrm{SO}_{4 m}}\left(\Theta_{\tau, 4 m}\right)=\left(2^{2 m}\right)$.

The definition of this notation and the proof of a similar proposition, for the symplectic group, can be found in [G3, Theorem 1] (see also [G3, p. 465, Case $(d)]$ ).

Let $\pi$ denote a generic irreducible cuspidal representation of $\mathrm{SO}_{2 m+1}(\mathbf{A})$. Following [G3], we consider the space of functions defined on $\mathrm{SO}_{2 m-1}(\mathbf{A})$ given by

$$
f(h)=\int_{\mathrm{SO}_{2 m+1}(F) \backslash \mathrm{SO}_{2 m+1}(\mathbf{A})} \varphi_{\pi}(g) \theta_{\tau, 4 m}((g, h)) d g .
$$

Here $\theta_{\tau, 4 m}$ is a vector in the space of the representation $\Theta_{\tau, 4 m}$. The function $f(h)$ defines an automorphic function of $\mathrm{SO}_{2 m-1}(\mathbf{A})$, and we denote by $\sigma$ the representation of $\mathrm{SO}_{2 m-1}(\mathbf{A})$ generated by the above functions. We have the following.

Theorem 4 ([G3, Theorem 2]). The representation $\sigma$ is a cuspidal representation of $\mathrm{SO}_{2 m-1}(\mathbf{A})$.

The details in [G3, Theorem 2] are given for the symplectic group but are the same for the orthogonal group.

We note that the constructed representation $\sigma$ could be 0 . However, there is a criterion for $\sigma$ to be generic, which in particular implies that $\sigma$ is nonzero.

ThEOREM 5 ([G3, Theorem 6$])$. The representation $\sigma$ is a generic representation if and only if the representation $\pi$ is an endoscopic representation with respect to $\tau$ and $\sigma$.

The sketch of the proof is as follows. A computation of the Whittaker coefficient of $\sigma$ implies that it is not 0 for some choice of data if and only if integral (2) is not 0 for some choice of data. But as argued in [G3], the nonvanishing of this period integral is equivalent to the fact that $\pi$ is 
endoscopic with respect to $\tau$ and a certain automorphic representation of $\mathrm{SO}_{2 m-1}(\mathbf{A})$. If $\sigma$ is not 0 , then the period integral

$$
\begin{aligned}
& \int_{\mathrm{SO}_{2 m+1}(F) \backslash \mathrm{SO}_{2 m+1}(\mathbf{A})} \int_{\mathrm{SO}_{2 m-1}(F) \backslash \mathrm{SO}_{2 m-1}(\mathbf{A})} \varphi_{\pi}(g) \\
& \times \varphi_{\sigma}(h) \theta_{\tau, 4 m}((g, h)) d h d g
\end{aligned}
$$

is not 0 for some choice of data. But then, a similar argument given in [G3, Sections 4, 5] or in the last section of [G1] proves that $\pi$ is endoscopic with respect to $\tau$ and $\sigma$.

Finally, we need to study a certain Fourier coefficient of $\Theta_{\tau, 4 m}$. Let $L_{2}$ denote the unipotent radical of the standard parabolic subgroup of $\mathrm{SO}_{4 m}$ whose Levi part is $\mathrm{GL}_{1}^{2} \times \mathrm{SO}_{4 m-4}$. We define a character $\psi_{L_{2}}$ on this unipotent group as follows. For $l=\left(l_{i, j}\right) \in L_{2}$, define $\psi_{L_{2}}(l)=\psi\left(l_{1,2}\right)$. For $g \in \mathrm{SO}_{4 m-4}(\mathbf{A})$, the functions

$$
g \mapsto \int_{L_{2}(F) \backslash L_{2}(\mathbf{A})} \theta_{\tau, 4 m}(l g) \psi_{L_{2}}(l) d l
$$

are automorphic functions of $\mathrm{SO}_{4 m-4}(\mathbf{A})$. We have the following.

Proposition 2. The representation of $\mathrm{SO}_{4 m-4}(\mathbf{A})$ generated by all the above functions is $\Theta_{\tau, 4 m-4}$.

The proof of this proposition follows from the definition of $\Theta_{\tau, 4 m}$ as a multiresidue of a certain Eisenstein series. See [G3, Proposition 1] for some details in a similar case done for the symplectic group.

Acknowledgment. We would like to thank the referee for helpful comments.

\section{REFERENCES}

[A] J. Arthur, The endoscopic classification of representations: Orthogonal and symplectic groups, preprint, 2011.

[CKPS] J. W. Cogdell, H. Kim, I. I. Piatetski-Shapiro, and F. Shahidi, Functoriality for the classical groups, Publ. Math. Inst. Hautes Études Sci. 99 (2004), 163-233.

[GS] W. T. Gan and G. Savin, Real and global lifts from $\mathrm{PGL}_{3}$ to $G_{2}$, Int. Math. Res. Not. IMRN 50 (2003), 2699-2724.

[G1] D. Ginzburg, A construction of CAP representations for classical groups, Int. Math. Res. Not. IMRN 20 (2003), 1123-1140.

[G2] Certain conjectures relating unipotent orbits to automorphic representations, Israel J. Math. 151 (2006), 323-355. 
[G3] - Endoscopic lifting in classical groups and poles of tensor L-functions, Duke Math. J. 141 (2008), 447-503.

[GRS] D. Ginzburg, S. Rallis, and D. Soudry, The Descent Map from Automorphic Representations of $\mathrm{GL}(n)$ to Classical Groups, World Sci., Hackensack, N.J., 2011.

[Ich] A. Ichino, On the regularized Siegel-Weil formula, J. Reine Angew. Math. 539 (2001), 201-234.

[Ik] T. Ikeda, On the theory of Jacobi forms and Fourier-Jacobi coefficients of Eisenstein series, Kyoto J. Math. 34 (1994), 615-636.

[J] D. Jiang, On the fundamental automorphic L-functions of $\mathrm{SO}(2 n+1)$, Int. Math. Res. Not. IMRN 2006, Art. ID 64069.

[JQ] D. Jiang and Y.-J. Qin, Residues of Eisenstein series and generalized Shalika models for $\mathrm{SO}(4 n)$, J. Ramanujan Math. Soc. 22 (2007), 101-133.

[JS] D. Jiang and D. Soudry, On the genericity of cuspidal automorphic forms of $\mathrm{SO}_{2 n+1}$, II, Compos. Math 143 (2007), 721-748.

[KR] S. S. Kudla and S. Rallis, A regularized Siegel-Weil formula: The first term identity, Ann. of Math. (2) 140 (1994), 1-80.

[S] D. Soudry, Rankin-Selberg integrals, the descent method, and Langlands functoriality, Int. Congr. Math. 2, Eur. Math. Soc., Zürich, 2006, 1311-1325.

David Ginzburg

School of Mathematical Sciences

Sackler Faculty of Exact Sciences

Tel-Aviv University

Israel 69978

ginzburg@post.tau.ac.il

Dihua Jiang

School of Mathematics

University of Minnesota

Minneapolis, Minnesota 55455

USA

dhjiang@math.umn.edu 